University of Nebraska - Lincoln

DigitalCommons@University of Nebraska - Lincoln

10-8-2001

\title{
Temporary anion states of selected amino acids
}

\author{
Kayvan Aflatooni \\ University of Nebraska-Lincoln, kaflatoo@fhsu.edu \\ B. Hitt \\ University of Nebraska-Lincoln \\ Gordon A. Gallup \\ University of Nebraska-Lincoln, ggallup1@unl.edu \\ Paul Burrow \\ University of Nebraska-Lincoln, pburrow1@unl.edu
}

Follow this and additional works at: https://digitalcommons.unl.edu/physicsburrow

Part of the Physics Commons

Aflatooni, Kayvan; Hitt, B.; Gallup, Gordon A.; and Burrow, Paul, "Temporary anion states of selected amino acids" (2001). Paul Burrow Publications. 8.

https://digitalcommons.unl.edu/physicsburrow/8

This Article is brought to you for free and open access by the Research Papers in Physics and Astronomy at DigitalCommons@University of Nebraska - Lincoln. It has been accepted for inclusion in Paul Burrow Publications by an authorized administrator of DigitalCommons@University of Nebraska - Lincoln. 


\title{
Temporary anion states of selected amino acids
}

\author{
K. Aflatooni, ${ }^{\text {a) }}$ B. Hitt, ${ }^{\text {b) }}$ G. A. Gallup, and P. D. Burrow \\ Department of Physics and Astronomy, University of Nebraska-Lincoln, Lincoln, Nebraska 68588-0111
}

(Received 29 May 2001; accepted 27 July 2001)

\begin{abstract}
Vertical attachment energies for the formation of low-lying temporary anion states of glycine, alanine, phenylalanine, tryptophan, and proline in the gas phase are reported using electron transmission spectroscopy. Electron attachment into the empty $\pi^{*}$ orbital of the $-\mathrm{COOH}$ group was observed in all the compounds. Temporary anion states associated with the side groups in phenylalanine and tryptophan are found to be stabilized with respect to those in the reference compounds toluene and indole, respectively, by approximately $0.2 \mathrm{eV}$. We attribute this to electrostatic effects and explore, using simple theoretical models, the extent to which such anion states could be further stabilized if these amino acids were in zwitterionic form. (C) 2001 American Institute of Physics. [DOI: 10.1063/1.1404147]
\end{abstract}

\section{INTRODUCTION}

The energy required to attach an electron into the lowest unoccupied molecular orbital (LUMO) of a molecule in the gas phase is a fundamental property that bears on numerous chemical processes related to the ease with which the molecule may be reduced. Determined at the equilibrium geometry of the neutral molecule, this quantity is called the vertical attachment energy (VAE), and its value provides a reference point from which the effects of solvation or other changes in the molecular environment may be studied. ${ }^{1}$ Furthermore, the gas phase value gives a benchmark for calibration of theoretical calculations that do not attempt to include solvent effects.

For molecules in which $\mathrm{VAE}>0$, the resulting anion states are short-lived and decay primarily by ejection of the electron back into the continuum. Low energy electron scattering techniques such as electron transmission spectroscopy $($ ETS $),{ }^{2}$ in which the anion states appear as resonances in the scattering cross section, are therefore natural and convenient means to locate these transient states. ${ }^{3,4}$ The valence anion states of the most fundamental biological constituents have received little attention in the gas phase using such methods. Temporary anion states in the four DNA bases and uracil, for example, have been reported only recently. ${ }^{5}$ This work showed that the lowest-lying valence anion states of the latter molecules are not stable at the equilibrium geometries of the neutrals and provided the VAEs associated with filling the LUMOs and several higher lying empty orbitals.

In the present work, we extend such measurements to several of the amino acids, the fundamental constituents of proteins. The experimental difficulties of working with the amino acids in the gas phase, namely their tendency to thermally decompose, are well known from earlier photoelectron spectroscopy (PES) studies. ${ }^{6}$ As a general guide, we find that ETS can be used in most molecules for which PES has been

${ }^{a)}$ Current address: Department of Physics, Fort Hays State University, Hays, KS 67601-4099.

${ }^{b)}$ Current address: J. A. Woollam Co., Lincoln, NE. carried out. In this study, we report results for glycine, alanine, phenylalanine, tryptophan, and proline. Data in cysteine are also presented but are compromised in part by signals from $\mathrm{CO}_{2}$, a decomposition product. To support our anion assignments, we also include the results of quantumchemical calculations of the lower virtual orbital energies of these amino acids.

\section{EXPERIMENT}

The ETS technique has been amply discussed in the literature along with its applications to complex molecules. ${ }^{2-4,7}$ Briefly, an energy-selected electron beam is directed through a gas cell containing a sufficient density of the target compound to partially attenuate the beam. The scattered electrons are rejected at a retarding electrode following the collision region, and the transmitted current, comprising the unscattered electrons, is collected. A sharp dip in this current as a function of electron energy signals the presence of a peak in the scattering cross section arising from the formation of a temporary anion state. To accentuate this structure, the energy of the electrons is modulated in the collision cell with a small ac voltage and the derivative of the transmitted current with respect to energy is acquired using a synchronous detector. ${ }^{2}$ Each "resonance" is therefore characterized by a minimum and a maximum in the derivative signal. The energy of the midpoint between these is assigned to the VAE.

In the present work, the collision cell and an oven source containing the compounds were separately temperature controlled. To minimize problems with surface charging of the entrance and exit apertures, the collision cell was generally kept at a higher temperature than the oven. The amino acids were obtained from Aldrich with quoted purities of $99 \%$ or $99+\%$. Each was loaded into the oven without further treatment, the system was evacuated to its base pressure of $(3-4) \times 10^{-7}$ Torr, and the temperatures of the cell and oven slowly increased until attenuation of the electron beam was observed. The oven/collision cell temperatures $\left({ }^{\circ} \mathrm{C}\right)$ were approximately as follows: glycine $155 / 165$, alanine $152 /--$, phenylalanine $160 /--$, tryptophan $185 / 254$, proline $120 / 190$, and 
cysteine 136/145. (Unfortunately the collision cell temperatures for alanine and phenylalanine were not recorded.) Following measurements in each compound, the sample oven was cleaned and the apparatus baked until no background signal could be seen.

In their PES studies, Cannington and $\mathrm{Ham}^{6}$ observed the production of $\mathrm{CO}_{2}$ from thermal degradation of the $\mathrm{COOH}$ group to some degree in all the amino acids upon heating. In our case, the pronounced temporary anion state at $3.57 \mathrm{eV}$ in $\mathrm{CO}_{2}$ can be used as an indicator for this particular decomposition process. In the compounds reported here, however, $\mathrm{CO}_{2}$ was only observed in cysteine. We have no other means, in situ, to observe decomposition products. We note that ETS is not a particularly sensitive method for detecting small admixtures of impurities unless the structures they produce in the transmission spectra are quite narrow. The features we observe are sufficiently broad and their association with the reference molecules so strong that it seems unlikely that they result from impurities, save for the case of cysteine. In our unsuccessful measurement in asparagine, the only signal observed by ETS was that of $\mathrm{CO}_{2}$, indicating that the other decomposition products did not produce low-lying temporary anion states.

The VAEs of the amino acids were energy calibrated to within $\pm 0.05 \mathrm{eV}$ by admitting $\mathrm{N}_{2}$ to the cell and referring to well known features arising from the $\mathrm{N}_{2}^{-}$temporary anion. The electron energy resolution cannot be determined directly from our data since there are no known features in the spectra sufficiently sharp to test it on. Prior to heating the oven and cell, the beam "turn-on" indicates a full-width at halfmaximum of approximately $60-80 \mathrm{meV}$.

\section{THEORETICAL}

$A b$ initio SCF calculations were carried out using the GAMESS program. ${ }^{8}$ Geometries of the neutral molecules were optimized in the $6-31 \mathrm{G}^{*}$ basis set, and virtual orbital energies were obtained with the same basis set. It is well known that such orbital energies are in substantial disagreement with experimental VAEs in an absolute sense. The relative values, however, within families of related molecules, can be usefully employed. By scaling the virtual orbital energies to VAEs determined by ETS in a few prototypical molecules, predicted VAEs for other similar compounds can be derived. A number of schemes for scaling have been put forth. ${ }^{9}$ In the present work, we employ the one in our DNA studies, ${ }^{5}$ using as standards the measured VAEs of the lowest anion states of benzene, naphthalene, pyridine, and pyrimidine. We found that $\mathrm{VAE}=\left[\varepsilon_{v o}-2.5553\right] / 1.3749$, where $\varepsilon_{v o}$ is the calculated virtual orbital energy in $\mathrm{eV}$.

At the temperatures of our measurements, a mixture of conformers of the amino acids will be present. When treating molecules with many conformers, automatic geometry optimization programs, such as GAMESS, can arrive at various local energy minima depending upon the starting point of the optimization. On the other hand, initial geometry-building software, such as that incorporated in SPARTAN, ${ }^{10}$ uses some simple rules to make conformation choices. Except for glycine, where we studied different starting points, our results are those generated from the starting geometry produced by SPARTAN. The more detailed study carried out for glycine indicated that the VAEs for attachment into the $\pi^{*}-\mathrm{COOH}$ orbitals for the four lowest lying conformers, of eight studied, were spread by only $71 \mathrm{meV}$. This accuracy will be sufficient to support our anion assignments.

\section{RESULTS}

With unsaturated compounds, the dominant resonances appearing in electron scattering cross sections arise from temporary occupation of normally empty $\pi^{*}$ orbitals. In such compounds made up of first row elements, the empty $\sigma^{*}$ orbitals lie higher in energy and the associated anion states have shorter lifetimes and thus greater spreads in energy, making them harder to isolate and identify. In each of the amino acids therefore, we expect to observe a pronounced feature characteristic of the normally empty $\pi^{*}$ orbital of the $-\mathrm{COOH}$ group common to the compounds. If the side groups are also unsaturated, as in the case of phenylalanine and tryptophan, we expect additional low-lying $\pi^{*}$ anion states similar to those of toluene and indole, respectively. At higher energies, broad features associated with the $\sigma^{*}(\mathrm{C}-\mathrm{C}, \mathrm{C}-\mathrm{N}, \mathrm{C}-\mathrm{H})$ orbitals will appear, however, we will not attempt to assign these states.

Table I summarizes VAE and $\Delta E_{d p}$, the dip to peak energy separation of the derivative structure in the transmission spectrum, for each of the temporary anion states, together with the orbital attribution, and the calculated virtual and scaled orbital energies.

\section{Glycine and Alanine}

We begin with the simplest of the amino acids, having side groups of $-\mathrm{H}$ and $-\mathrm{CH}_{3}$, respectively, neither of which will introduce low-lying anion states. The ET spectra are shown in Fig. 1 along with that of $\mathrm{HCOOH}$, which we use as a reference molecule for the $-\mathrm{COOH}$ group, and $\mathrm{CO}_{2}$, which we include to indicate where this decomposition product would appear in the spectra. The vertical lines in each panel indicate the energies of the VAEs. ET spectra of $\mathrm{HCOOH}^{11}$ and $\mathrm{CO}_{2}$ (Ref. 12) taken close to ambient temperatures have been published previously and show weak vibrational structures. These are likely to be obscured at the higher temperatures used here because of the greater population of excited vibrational levels of the molecules.

The close similarity of the ET spectra of glycine and alanine to that of $\mathrm{HCOOH}$, with respect to the energies at which the features appear and their widths, $\Delta E_{d p}$, confirms the origin of these anion states to temporary occupation of the $\pi^{*}$ orbital of the $-\mathrm{COOH}$ group, in agreement with the theoretical results. No sign of structure owing to the presence of $\mathrm{CO}_{2}$ was observed.

\section{Phenylalanine}

Figure 2 shows the spectra of phenylalanine and toluene, a reference molecule for the side group. The spectrum of the latter has been reported earlier with higher energy resolution. ${ }^{13}$ The major feature in the toluene spectrum near $1.21 \mathrm{eV}$ derives from the doubly degenerate $e_{2 u}\left(\pi_{1,2}^{*}\right)$ orbit- 
TABLE I. Measured vertical attachment energies, dip to peak widths, calculated virtual orbital energies in the 6-31G* basis set, and scaled virtual orbital energies as described in the text. All values are in units of electronvolts.

\begin{tabular}{llcccc}
\hline \hline Amino acid & \multicolumn{1}{c}{ Molecular orbital } & VAE & $\Delta E_{d p}$ & VOE & Scaled VOE \\
\hline Glycine & $\pi^{*}(-\mathrm{COOH})$ & 1.93 & 0.96 & 5.1171 & 1.863 \\
Alanine & $\pi^{*}(-\mathrm{COOH})$ & 1.80 & 0.93 & 4.9215 & 1.72 \\
Phenylalanine & $\pi_{1,2}^{*}(-\phi)$ & 0.87 & 0.57 & $3.8074,3.9013$ & $0.911,0.979$ \\
& $\pi_{3}^{*}(-\mathrm{COOH})$ & 1.85 & $>0.33^{\mathrm{a}}$ & 5.1226 & 1.867 \\
\multirow{2}{*}{ Tryptophan } & $\pi_{4}^{*}(-\phi)$ & 4.51 & 1.00 & & \\
& $\pi_{1}^{*}(\mathrm{s.g} .)^{\mathrm{b}}$ & 0.68 & 0.37 & 3.7269 & 0.852 \\
& $\pi_{2}^{*}(\mathrm{s.g}),. \pi_{3}^{*}(-\mathrm{COOH})$ & 1.60 & 0.49 & $4.8739,5.1773$ & $1.686,1.907$ \\
Proline & $\pi_{4}^{*}$ & 2.50 & 0.39 & 6.1090 & 2.585 \\
& $\pi^{*}(-\mathrm{COOH})$ & 1.91 & 1.03 & 5.3688 & 2.0463 \\
Cysteine & $\pi^{*}(-\mathrm{COOH})$ & 5.4 & 2.37 & & \\
& $\left(\mathrm{CO}_{2}\right.$ decomp. $)$ & 1.98 & 0.91 & 4.3277 & 1.289 \\
& & 3.59 & 0.92 & &
\end{tabular}

Reference molecules

\begin{tabular}{lllccc}
\hline $\mathrm{HCOOH}$ & $\pi^{*}(-\mathrm{COOH})$ & 1.73 & 0.82 & 5.1082 & 1.857 \\
$\mathrm{CO}_{2}$ & & 3.58 & 0.93 & & \\
Toluene & $\pi_{1,2}^{*}$ & 1.21 & 0.66 & $4.0828,4.1394$ & $1.111,1.149$ \\
& $\pi_{3}^{*}$ & 4.80 & 0.72 & & \\
Indole & $\pi_{1}^{*}$ & 0.90 & 0.33 & 3.7615 & 0.877 \\
& $\pi_{2}^{*}$ & 1.85 & 0.40 & 4.9746 & 1.7603 \\
& $\pi_{3}^{*}$ & 2.71 & 0.56 & 6.2475 & 2.685 \\
Pyrrolidine & $\sigma^{*} ?$ & 1.77 & 1.33 & $6.1253 ?$ & \\
& $\sigma^{*}$ & 6.01 & 2.12 & & \\
\hline \hline
\end{tabular}

${ }^{\mathrm{a}}$ See text.

${ }^{\mathrm{b}}$ s.g. $=$ side group.

als of benzene. Although the additional structure between 1.25 and $1.65 \mathrm{eV}$ has the appearance of a separate anion state, the $\pi_{1,2}^{*}$ orbitals in toluene are believed to be spaced by only $33 \mathrm{meV} .{ }^{14}$ Because similar structure exists in the benzene spectrum, the origin of the structure in toluene appears not to arise from large splitting of the two orbitals. The upper feature at $4.8 \mathrm{eV}$ in toluene derives from the benzene $b_{2 g}\left(\pi_{3}^{*}\right)$ orbital.

Each of the "toluene" resonances is observed in the phenylalanine spectrum, along with that from the $-\mathrm{COOH}$ group near $1.85 \mathrm{eV}$. The latter is overlapped by the contribution from the $1.1 \mathrm{eV}$ structure owing to the side group, and its energy cannot be determined as precisely. No sign of the $\mathrm{CO}_{2}$ decomposition product is observed. We note that the anion states arising from the side group are each stabilized relative to those in toluene, the lower (quasi-degenerate) state by $0.23 \mathrm{eV}$ and the upper by approximately $0.37 \mathrm{eV}$.

\section{Tryptophan}

Figure 3 shows the ET spectra of tryptophan and indole, the reference molecule for its side group. The spectra are quite similar, although the anion states associated with the side group of tryptophan are stabilized by about $0.2 \mathrm{eV}$ relative to those in indole. The tryptophan anion state associated with the $-\mathrm{COOH}$ group, normally expected to lie near 1.8 $\mathrm{eV}$, does not appear as a separate feature. This resonance is likely to be obscured by the second anion state arising from the side group. In support of this, we note that the second

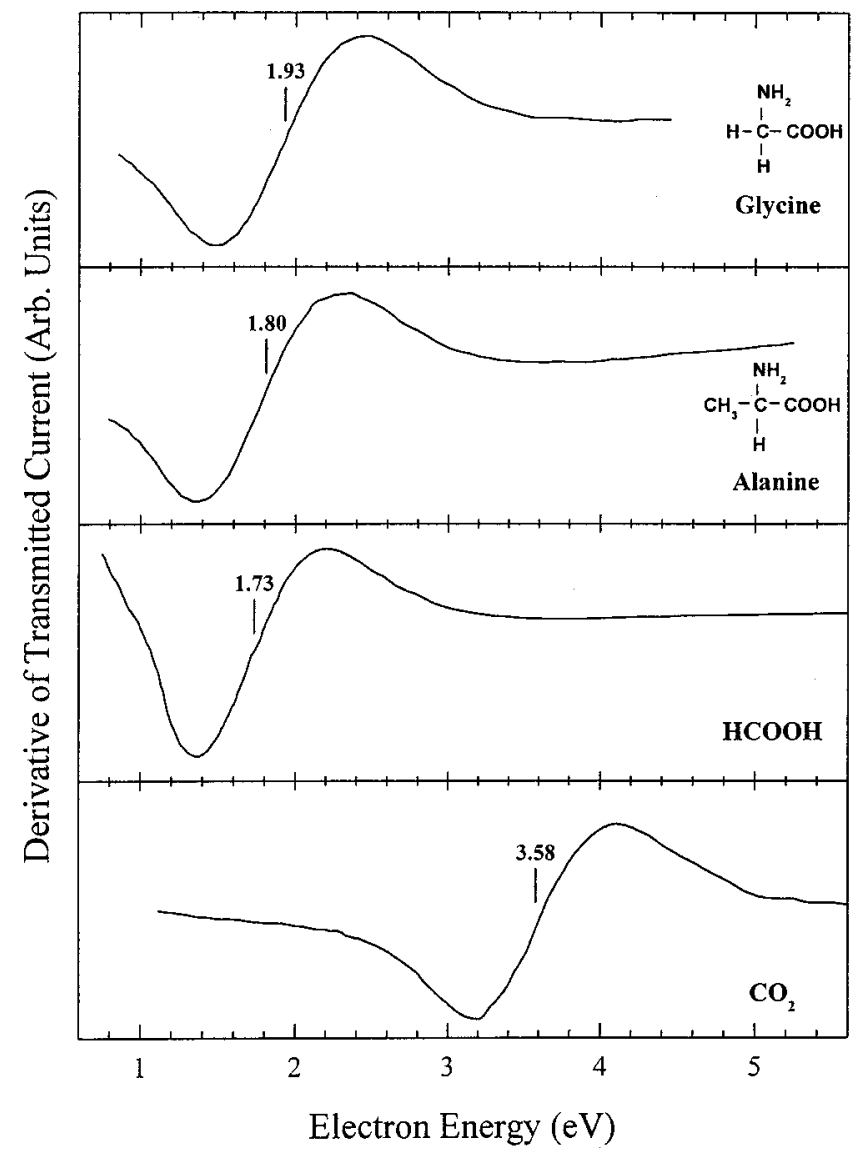

FIG. 1. The derivative with respect to energy of transmitted electron current as a function of electron energy in glycine, alanine, $\mathrm{HCOOH}$, and $\mathrm{CO}_{2}$. 


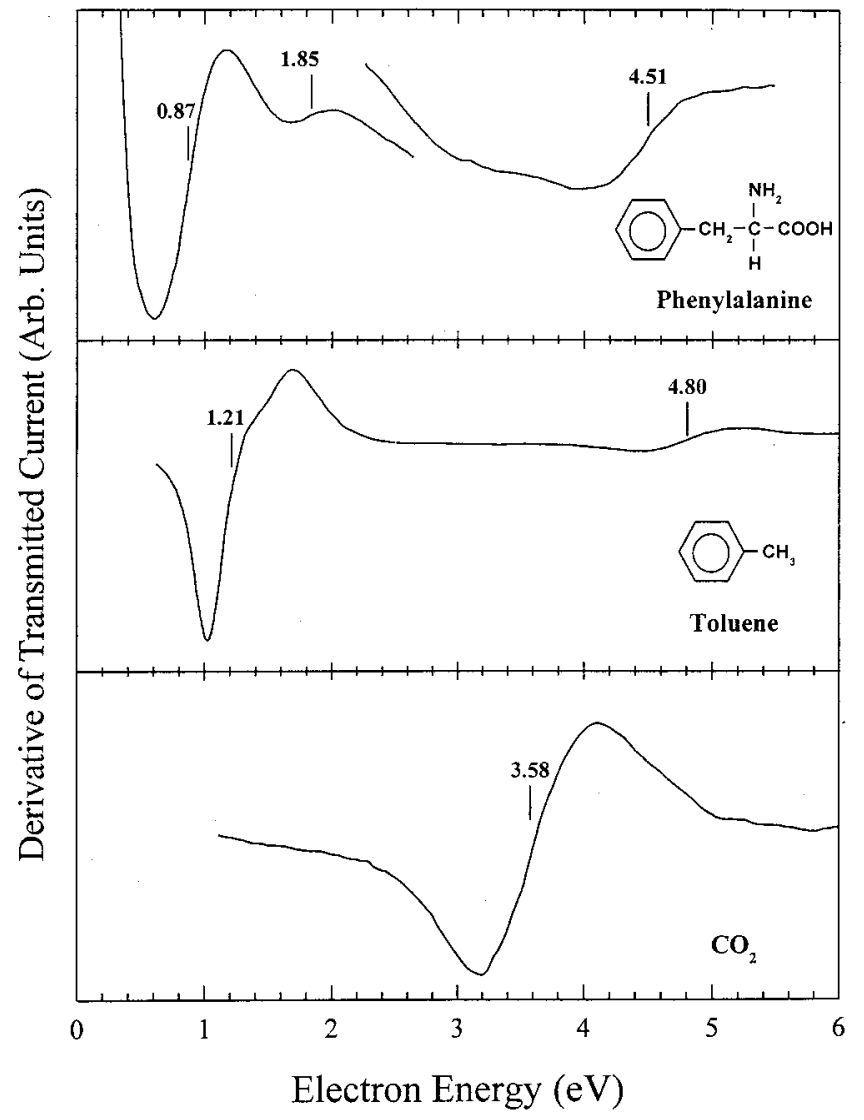

FIG. 2. As in Fig. 1, for phenylalanine, toluene, and $\mathrm{CO}_{2}$.

resonance in tryptophan is considerably larger in magnitude relative to the first resonance than it is in indole, consistent with the overlap of two anion states.

A feature in the tryptophan spectrum occurs near $3.5 \mathrm{eV}$, close to the value expected from a $\mathrm{CO}_{2}$ impurity. However, the width of this resonance is narrower than that of $\mathrm{CO}_{2}$, and it is likely to be associated with a core-excited anion state in tryptophan.

\section{Proline}

Figure 4 shows the ET spectra of proline and a reference molecule, pyrrolidine. Proline is an anomalous amino acid in that the side group consists of three $\mathrm{CH}_{2}$ groups added in cyclic fashion to the $\mathrm{CH}-\mathrm{NH}$ structure. The resulting fivemembered ring is saturated, and we would not expect a lowlying anion state to arise from it. Surprisingly, the pyrrolidine reference spectrum clearly shows a feature at $1.77 \mathrm{eV}$ in addition to a higher lying resonance at $6.0 \mathrm{eV}$. The assignment of the lower feature is not clear, and our virtual orbital calculations do not yield such a low-lying anion state. We cannot rule out the possibility that the structure in the ET spectrum arises from an impurity, although the feature and a similar one in cyclopentane have been observed independently elsewhere. ${ }^{15}$

The proline spectrum shows its most prominent structure at $1.91 \mathrm{eV}$. The width and profile of this feature match very well those of the $-\mathrm{COOH} \pi^{*}$ resonances in glycine and alanine, and we attribute it primarily to this orbital in proline

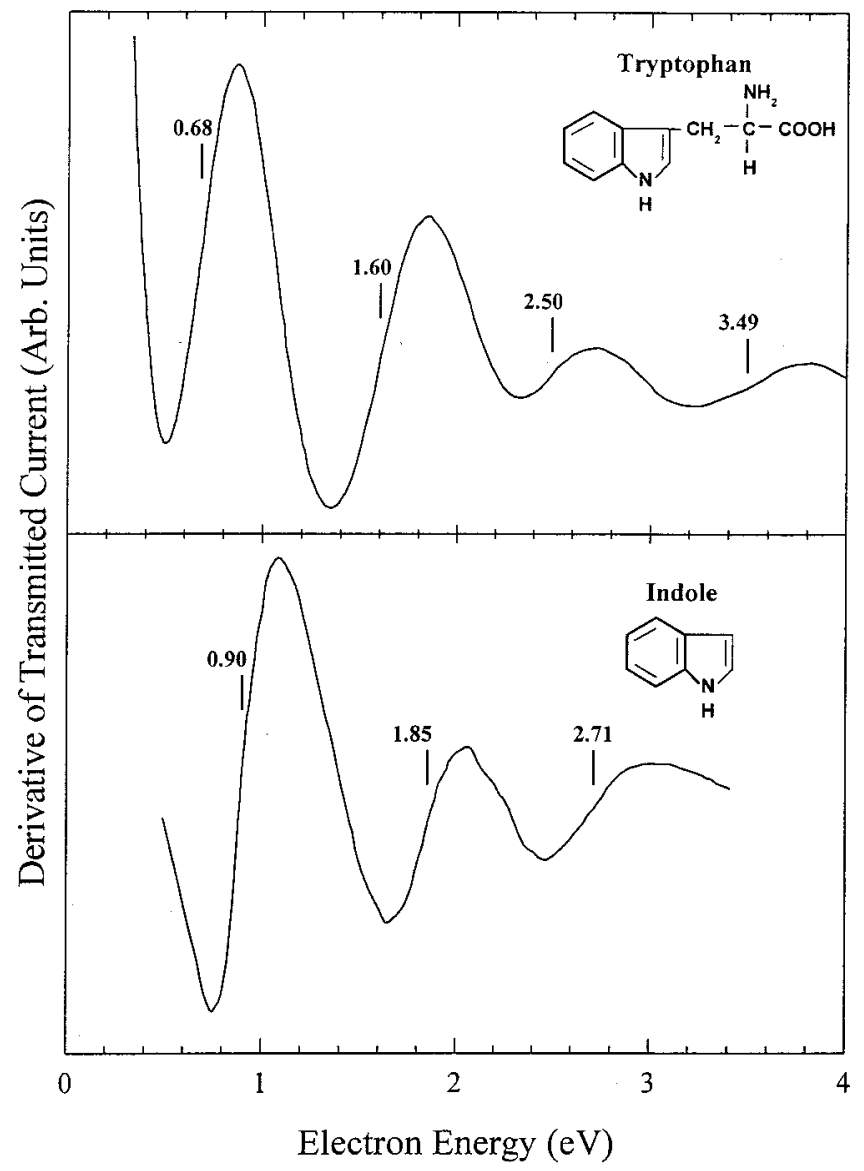

FIG. 3. As in Fig. 1, for tryptophan and indole.

but note that it could well overlap and obscure a smaller and broader contribution from the five-membered ring. No evidence for thermal production of $\mathrm{CO}_{2}$ is observed.

\section{Cysteine}

Of several other amino acids that were attempted, including cysteine, histadine, asparagine, and arginine, none gave ET spectra that were free of $\mathrm{CO}_{2}$. We present in Fig. 5 only the results in cysteine as an example of the least problematic of these. The lower resonance is entirely consistent with that arising from the $-\mathrm{COOH}$ group with respect to energy and width. The $-\mathrm{CH}_{2} \mathrm{SH}$ side group is best modeled by $\mathrm{CH}_{3} \mathrm{SH}$. ETS studies in this compound were carried out by Dezarnaud et al. ${ }^{16}$ who reported a very broad feature $\left(\Delta E_{d p} \approx 2.5 \mathrm{eV}\right)$ centered at 2.85 and attributed to a $\sigma^{*}(\mathrm{C}-\mathrm{S})$ orbital. These characteristics are not in good agreement with the upper resonance observed in cysteine. The properties of the upper feature, however, are entirely consistent with those of $\mathrm{CO}_{2}$. Since thermal production of $\mathrm{CO}_{2}$ is done at the cost of the $-\mathrm{COOH}$ group, the presence of both resonances seems to imply that comparable amounts of the parent compound as well as the decomposition products could be present. We suggest that the anion state associated with the $\sigma^{*}(\mathrm{C}-\mathrm{S})$ orbital is so weak and broad that it is obscured by the $-\mathrm{COOH}$ and $\mathrm{CO}_{2}$ resonances. 


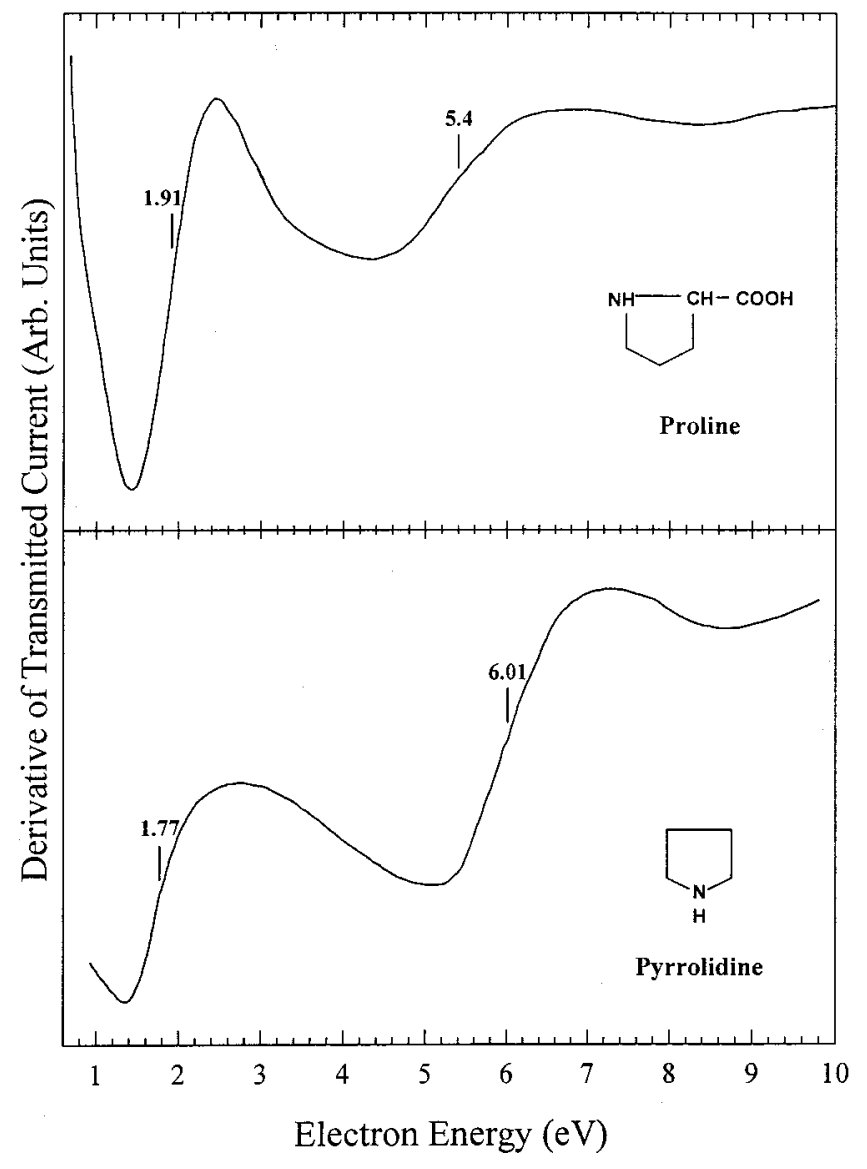

FIG. 4. As in Fig. 1, for proline and pyrrolidine.

\section{DISCUSSION}

VAEs for the amino acids and the reference molecules studied here are in good agreement with the scaled orbital energies, considering the limited exploration of the various conformers. The only puzzle is the low-lying resonance observed in pyrrolidine, which warrants further study, both experimentally and theoretically.

A noteworthy feature of the results we have presented here is the roughly $0.2 \mathrm{eV}$ stabilization of the $\pi^{*}$ anion states associated with the side groups of the amino acids with respect to the corresponding states in the reference molecules. A possible source of this stabilization is the electric dipole field of the portion of the amino acid away from the side group. The magnitude of this stabilization encouraged us to consider how the energies of the $\pi^{*}$ anion states of the side groups would be modified by the large dipole moment present in the zwitterionic form of the amino acids. In this structure, the $-\mathrm{COOH}$ group loses a proton to become $-\mathrm{CO}_{2}^{-}$ and the $-\mathrm{NH}_{2}$ group becomes $-\mathrm{NH}_{3}^{+}$, producing a very large local dipole moment that, if oriented correctly, could substantially stabilize the LUMOs of phenylalanine and tryptophan. We next examine at a crude theoretical level the possible extent of this stabilization in phenylalanine.

There is no firm evidence that stable zwitterions of the amino acids can occur in the gas phase. Nevertheless, we may construct a hypothetical zwitterion species of phenylalanine using appropriate bond distances and angles from

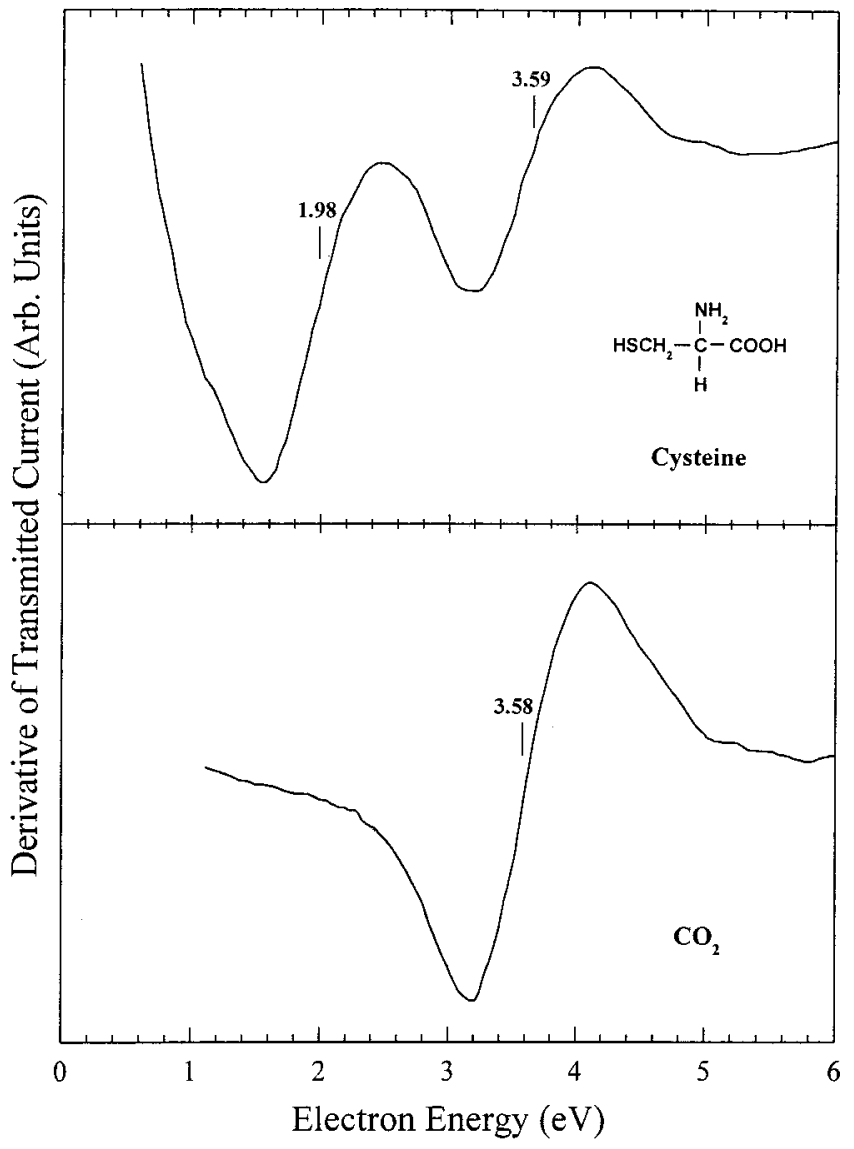

FIG. 5. As in Fig. 1, for cysteine and $\mathrm{CO}_{2}$.

$\mathrm{N}$-protonated and O-deprotonated phenylalanine. With a 6-31G* basis, this hypothetical structure cannot be subjected to an unconstrained geometry optimization without having one of the protons in the $-\mathrm{NH}_{3}^{+}$group migrate to an oxygen on $-\mathrm{CO}_{2}^{-}$, returning the structure to the normal gaseous form. Constraining some of the positions, however, allows us to carry out a computer investigation of the effect of the large dipole moment of the zwitterionic group on the energies of the $\pi^{*}$ resonances in the phenyl ring. As a first test, the $\mathrm{CHNH}_{3}^{+}-\mathrm{CO}_{2}^{-}$fragment of the molecule, which we label $A$, was rotated rigidly about the single bond attaching it to the $\mathrm{CH}_{2}-\mathrm{C}_{6} \mathrm{H}_{6}$ fragment containing the benzene ring. The overall dipole moment varied over a range of about 1 Debye around the value of 12.7 Debye as A executed a $360^{\circ}$ rotation. As expected, the electric potential of the dipole at the benzene ring changes sign during the rotation, producing an approximately sinusoidal variation in the LUMO energy as a function of angle.

The minimum LUMO energy occurs at an angle only a few degrees away from the geometry at which the total energy is minimized. At this latter geometry, we find that the LUMO of the phenylalanine "zwitterion" has a calculated virtual orbital energy of $\varepsilon_{v o}=2.7353 \mathrm{eV}$. Using the scaling procedure given earlier, this corresponds to a VAE of 0.13 $\mathrm{eV}$; that is, the lowest valence anion state is vertically unstable by only $0.13 \mathrm{eV}$, a stabilization of $0.74 \mathrm{eV}$ with respect to its value in normal phenylalanine. Similar calculations 
were not carried out for tryptophan, however, considering that its first VAE is approximately $0.2 \mathrm{eV}$ lower than that of phenylalanine, it is not unreasonable to expect that in its zwitterionic form, even a stable valence anion could exist.

Given the uncertainties in the restricted geometry of the zwitterion structure employed here, it would be a mistake to overinterpret this approximate treatment. Nevertheless, the results are suggestive that stable anionic states of the tryptophan and possibly the phenylalanine zwitterions could exist. Substantial electrostatic effects in the binding of an electron in the zwitterionic anion of methylated pyridinium-3,5dicarboxylate have been observed by Wang et al. ${ }^{17}$ Although no experiments showing the existence of neutral zwitterions of the amino acids in the gas phase are known to us, it seems likely that such species will be formed in clusters in which the acid is partially solvated by water molecules. In such an environment, the solvent molecules would be expected to additionally stabilize the proposed valence anionic zwitterions, although it must be said that a concomitant reduction of the dipole moment could also take place that would be destabilizing. If such anion states are indeed stable, they might appear energetically near the well known dipole-bound anion states generated by molecules with such large dipole moments, but should be distinguishable from them by valencelike structure in the photodetachment spectrum.

\section{ACKNOWLEDGMENTS}

This work was supported by the National Science Foundation. One of the authors (B.H.) was an undergraduate participant in this research.

${ }^{1}$ See, for example, S. A. Lyapustina, S. Xu, J. M. Nilles, and K. H. Bowen, Jr., J. Chem. Phys. 112, 6643 (2000); J. Schiedt, W. J. Knott, K. Le Barbu, E. W. Schlag, and R. Weinkauf, ibid. 113, 9470 (2000).

${ }^{2}$ L. Sanche and G. J. Schulz, Phys. Rev. A 5, 1672 (1972).

${ }^{3}$ K. D. Jordan and P. D. Burrow, Acc. Chem. Res. 11, 341 (1978).

${ }^{4}$ K. D. Jordan and P. D. Burrow, Chem. Rev. 87, 557 (1987).

${ }^{5}$ K. Aflatooni, G. A. Gallup, and P. D. Burrow, J. Phys. Chem. A 102, 6205 (1998)

${ }^{6}$ P. H. Cannington and S. Ham, J. Electron Spectrosc. Relat. Phenom. 15, 79 (1979).

${ }^{7}$ A. Modelli, Trends Chem. Phys. 6, 57 (1997).

${ }^{8}$ M. W. Schmidt, K. K. Baldridge, J. A. Boatz, S. T. Elbert, M. S. Gordon, J. H. Jensen, S. Koseki, N. Matsunaga, K. A. Nguyen, S. J. Su, T. L. Windus, M. Dupuis, and J. A. Montgomery, J. Comput. Chem. 14, 1347 (1993). See also: http://www.msg.ameslab.gov/GAMEss.html

${ }^{9}$ See, for example, D. Chen and G. A. Gallup, J. Chem. Phys. 93, 8893 (1990); S. W. Staley and J. T. Strnad, J. Phys. Chem. 98, 116 (1994).

${ }^{10}$ SPARTAN, Wavefunction, Inc. 18401 Von Karman Avenue, Suite 370, Irvine, CA 92715.

${ }^{11}$ M. Tronc, M. Allan, and F. Edard, Proceedings of the ICPEAC 1987, p. 335.

${ }^{12}$ L. Sanche and G. J. Schulz, J. Chem. Phys. 58, 479 (1973).

${ }^{13}$ K. D. Jordan, J. A. Michejda, and P. D. Burrow, J. Am. Chem. Soc. 98, 1295 (1976).

${ }^{14}$ J. S. Alper and R. J. Silbey, J. Chem. Phys. 52, 569 (1970).

${ }^{15} \mathrm{~A}$. Modelli (private communication).

${ }^{16}$ C. Dezarnaud, M. Tronc, and A. Modelli, Chem. Phys. 156, 129 (1991).

${ }^{17}$ X-B. Wang, K. M. Broadus, L-S. Wang, and S. R. Kass, J. Am. Chem. Soc. 122, 8305 (2000). 